\title{
The nature of hemispheric specialization for prosody perception
}

\author{
Jurriaan Witteman • Katharina S. Goerlich-Dobre • \\ Sander Martens • André Aleman • \\ Vincent J. Van Heuven • Niels O. Schiller
}

Published online: 11 February 2014

(C) Psychonomic Society, Inc. 2014

\begin{abstract}
Recent evidence suggests a relative righthemispheric specialization for emotional prosody perception, whereas linguistic prosody perception is under bilateral control. It is still unknown, however, how the hemispheric specialization for prosody perception might arise. Two main hypotheses have been put forward. Cue-dependent hypotheses, on the one hand, propose that hemispheric specialization is driven by specialization for the non-prosody-specific processing of acoustic cues. The functional lateralization hypothesis, on the other hand, proposes that hemispheric specialization is dependent on the communicative function of prosody, with emotional and linguistic prosody processing being lateralized to the right and left hemispheres, respectively. In the present study, the functional lateralization hypothesis of prosody perception was systematically tested by instructing
\end{abstract}

Electronic supplementary material The online version of this article (doi:10.3758/s13415-014-0255-1) contains supplementary material, which is available to authorized users.

J. Witteman • V. J. Van Heuven • N. O. Schiller

Leiden Institute for Brain and Cognition, Leiden University,

Leiden, The Netherlands

J. Witteman • V. J. Van Heuven • N. O. Schiller

Leiden University Centre for Linguistics, Leiden University,

Leiden, The Netherlands

K. S. Goerlich-Dobre · S. Martens · A. Aleman Neuroimaging Center, Department of Neuroscience, University Medical Center Groningen, University of Groningen, Groningen, The Netherlands

K. S. Goerlich-Dobre

Department of Psychiatry, Psychotherapy, and Psychosomatics, Medical School, RWTH Aachen University, Aachen, Germany

J. Witteman $(\bowtie)$

Leiden University, Cleveringaplaats 1 (Lipsius Building),

Room 104, 2311 BD Leiden, The Netherlands

e-mail: witteman.j@gmail.com one group of participants to evaluate the emotional prosody, and another group the linguistic prosody dimension of bidimensional prosodic stimuli in a dichotic-listening paradigm, while event-related potentials were recorded. The results showed that the right-ear advantage was associated with decreased latencies for an early negativity in the contralateral hemisphere. No evidence was found for functional lateralization. These findings suggest that functional lateralization effects for prosody perception are small and support the structural model of dichotic listening.

Keywords Prosody $\cdot$ Emotional $\cdot$ Linguistic $\cdot$ Lateralization Dichotic listening $\cdot$ ERP

Speech prosody is an important means to convey emotions (e.g., indicating whether the speaker is angry or sad) and linguistic structure (e.g., signifying a statement or a question). A longstanding question in the neuropsychological literature has been whether there is hemispheric specialization for the perception of prosody. Recent meta-analytic evidence suggests a relative right-hemispheric specialization for emotional prosody perception, whereas the processing of linguistic prosody seems to be controlled bilaterally (Witteman, van IJzendoorn, van de Velde, van Heuven, \& Schiller, 2011).

However, the nature of this relative right-hemispheric specialization for emotional prosody is currently unknown. Two mechanisms have been proposed as to how hemispheric specialization for prosody perception might arise. On the one hand, cue-dependent lateralization hypotheses propose that right-hemisphere specialization for emotional prosody perception can be explained by a (non-prosody-specific) advantage of the right hemisphere for early acoustic processing, such as spectral processing (Van Lancker \& Sidtis, 1992), since spectral parameters appear to be particularly important for decoding emotional prosody (Scherer, 2003). Indeed, a recent 
meta-analysis of the neuroimaging literature of emotional prosody perception revealed preliminary evidence for relative right-hemispheric specialization of the primary and secondary auditory cortex (Witteman, Van Heuven, \& Schiller, 2012), which could be interpreted as (indirect) support for the cuedependent lateralization hypothesis.

On the other hand, the functional lateralization hypothesis posits that the right hemisphere is specialized for the processing of the emotional communicative function of emotional prosody (Van Lancker, 1980), whereas the left hemisphere might be specialized for the processing of linguistic prosodic function. The dynamic dual-pathway model of Friederici and Alter (2004) further suggests that when linguistic prosody is more bound to segments (such as in the case of metrical stress), it is left lateralized, whereas linguistic prosody at the sentence level (such as boundary marking) is right lateralized. The goal of the present investigation was to systematically test whether functional lateralization occurs in prosody perception.

To comprehensively test the functional lateralization hypothesis, it is necessary to vary the communicative function of prosodic materials while keeping acoustics constant, and to observe whether the difference of activity between the hemispheres changes. Note that the acoustic and functional lateralization hypotheses are non-mutually-exclusive, and could represent different stages of the prosody perception process. Indeed, recent neural models of prosody perception have suggested that lateralization might be acoustically driven in an initial processing stage, but more semantically (functionally) driven in subsequent stages (Brück, Kreifelts, \& Wildgruber, 2011; Kotz \& Paulmann, 2011). To shed light on the issue of when in time functional lateralization arises, in the present event-related potential (ERP) study we systematically manipulated the function of prosody by instructing one group of participants to evaluate the emotional prosody dimension and a different group of participants to evaluate the linguistic prosody dimension of identical bidimensional stimuli.

Interestingly, Paulmann, Jessen, and Kotz (2012) recently reported such a direct comparison of emotional to linguistic prosody perception using the cross-splicing paradigm. A socalled "prosodic expectancy positivity" (PEP) was found that was more pronounced for emotional than for linguistic prosody expectancy violations, suggesting prioritized processing of emotional prosodic cues. However, the authors did not find task-driven effects, and suggested that the absence of such effects at the electrophysiological level might have been caused by a lack of statistical power to detect (presumably small) task effects, requiring the investigation of this issue using larger study samples. Furthermore, a within-subjects manipulation of task demands was employed, which required participants to switch between an emotional and a linguistic task set. As the authors pointed out, this procedure might have reduced task-driven differences in prosody processing, warranting a more extended investigation of this issue using a between-subjects manipulation of task demands.

The dichotic-listening (DL) paradigm is particularly suited to study hemispheric specialization in the auditory modality (Greenwald \& Jerger, 2003). In the divided-attention version of this paradigm, two different stimuli are presented to each ear. The participant has to divide attention over the auditory channels and react only to the target stimulus presented to one of the ears. It is generally agreed that in the DL situation, the ipsilateral projection of information from the ears to the cerebral hemispheres is inhibited, rendering auditory information from the ears primarily available to the contralateral hemisphere (see, e.g., Davidson \& Hugdahl, 1995). Therefore, if there were hemispheric specialization for the processing of an auditory stimulus, this would be observable as a performance advantage of the ear contralateral to the specialized hemisphere, as it has direct access to the specialized processing module (for a discussion of the exact mechanisms behind DL, see Grimshaw, Kwasny, Covell, \& Johnson, 2003).

Only one previous study has combined the DL paradigm with ERPs to study the lateralization of emotional prosody perception. Erhan, Borod, Tenke, and Bruder (1998) presented participants with dichotic pairs of nonsense syllables, each of which was spoken in one of seven emotional prosodic categories. Participants had to detect a prespecified emotional prosodic category as quickly as possible while the electroencephalogram (EEG) was recorded. Auditory target detection studies like these generally show an initial negativity followed by a positivity for targets as compared with nontargets (see, e.g., Fitzgerald \& Picton, 1983). In the case of the positivity, it has been demonstrated that its amplitude increases when target probability decreases, whereas for the earlier negativity, this effect is weak at best (Polich \& Bondurant, 1996). The positivity can be subdivided into the P3b component and the slow wave, which together have been termed the "late positive potential," or LPP (see, e.g., Briggs \& Martin, 2009). Erhan et al. indeed found an $\mathrm{N} 1$ and a sustained negativity, followed by a late positivity and a slow wave. Furthermore, at the behavioral level a left-ear advantage (LEA) was found for accuracy, in line with a right-hemispheric specialization for emotional prosody. The sustained negativity $(300-879 \mathrm{~ms}$ post-stimulus-onset) was identified as a potential electrophysiological marker of the behavioral ear advantage, hypothesized to reflect the emotional categorization process. However, although the relatively late latency of the component might be interpreted as reflecting fairly late (and therefore possibly functional) processing, strictly speaking, it is unclear whether this lateralized component reflected early acoustic lateralization, more abstract functional hemispheric specialization, or both, since the function of prosody was not manipulated independently of the acoustics (or vice versa). 
In the present ERP study, linguistic and emotional prosodic task demands were manipulated between subjects with comparably high statistical power, while keeping acoustics constant in a divided-attention dichotic auditory target detection paradigm. We predicted that, if there is functional lateralization of prosodic perception, the right ear should have an advantage for the linguistic prosody perception task, and the left ear an advantage for the emotional task. At the electrophysiological level, we hypothesized that the distribution of the N2 or LPP over the hemispheres might change correspondingly, demonstrating functional lateralization. Finally, we considered the possibility that an earlier component (reflecting acoustic processing) might also be sensitive to variation in task demands, reflecting top-down taskdependent (and possibly lateralized) modulation of earlier acoustic processing, as has been demonstrated previously (Sussman, Winkler, Huotilainen, Ritter, \& Näätänen, 2002).

\section{Method}

\section{Participants}

A total of 82 participants took part in the experiment. Five of the participants were excluded because of lower-than-chancelevel performance, and three due to noisy EEG data, resulting in a total of 74 participants: 41 (21 male, 20 female; mean age 23 years, range 18-37) for the emotional prosodic task, and 33 different participants (16 male, 17 female; mean age 23 years, range 19-36) for the linguistic prosodic task. The two groups did not differ in male:female ratio or age (for all: one-way analysis of variance, $F<1.01, p>$.5) All participants were right-handed as assessed with the Edinburgh Handedness Inventory (Oldfield, 1971), were native speakers of Dutch, and had normal or corrected-to-normal vision and no (neuro)psychiatric problems in the present or the past. Participants showing a mean interear hearing threshold difference greater than $12 \mathrm{~dB}$ on the $0.5-\mathrm{kHz}, 1-\mathrm{kHz}, 2-\mathrm{kHz}$, and $4-\mathrm{kHz}$ sinusoid tones were excluded from the study. Participants received $€ 20$ for their participation in the 2-h EEG session. Informed consent was obtained from all participants prior to the experiment. The study was approved by the local ethics committee and conducted in accordance with the Declaration of Helsinki.

\section{Materials}

Pseudowords (see the Appendix) with a bisyllabic structure were generated by randomly combining monosyllables that were composed of random combinations of an initial consonant, a vowel, and a final consonant. All of the pseudowords obeyed Dutch phonotactics and were checked to verify the absence of semantic content. All pseudowords were uttered with angry and sad prosody and with stress on the first or the second syllable by a professional actress and recorded at 16bit resolution and a $44.1-\mathrm{kHz}$ sampling rate in a soundproofed booth. Items were intensity normalized and had a mean duration of $750 \mathrm{~ms}$. In line with the previous literature (Cutler, 2005; Scherer, 2003), unstressed syllables differed from stressed syllables primarily in duration, whereas sad and angry prosody additionally differed in $\mathrm{F}_{0}, \mathrm{~F}_{0}$ variability, and variation in intensity (see Table 1). Note that the angry and sad items did not show large intensity differences, because the stimuli had been intensity-normalized. To verify the validity of the intended prosodic contrasts, a panel of five healthy volunteers classified each sad and angry prosodic stimulus (in addition to neutral, happy, and surprised prosodic stimuli that were not used in the present study) in a forced choice task and rated each item on a five-point typicality scale $(1=$ very atypical, 5 = very typical). Pseudowords were only selected if the emotional prosodic contrasts (angry and sad intonations) were classified correctly by at least four out of the five panel members and if they had a typicality rating of at least 3.5.

Evidence has indicated that increasing spectral overlap between the target and the competing stimulus increases the suppression of ipsilateral afferent routes from the ears to the cerebral hemispheres, which enhances ear advantages (Della Penna et al., 2007). Therefore, to maximize spectral overlap, a competing babble stimulus was created by selecting random small segments of speech of the actress and superimposing them onto each other. Dichotic stimuli were created by selecting a random sample of the babble stimulus with the exact same duration as the target stimulus and combining the two (with the target being presented in one channel and the babble stimulus in the other). This procedure ensured constant and high competition between the target stimulus and the distractor.

Two dichotic target detection tasks were created in which identical prosodic dichotic targets were presented. From the pool of validated dichotic stimuli, six that had sad prosody and stress on the first syllable served as the targets. For the emotional task, participants were instructed to press as quickly as possible when they heard a sad target. For the linguistic task, they were to respond as quickly as possible when targets had word-initial stress. Hence, identical stimuli served as the targets for both tasks, while only the task demands (emotional vs. linguistic decision) varied. Ten items with angry prosody and stress on the second syllable served as the nontargets in both target detection tasks. Additionally, four task-specific (nontarget) filler items were presented ten times to each ear but were not analyzed. For the emotional task, angry items with stress on the first syllable were added to prevent participants from using a linguistic strategy to detect emotional prosody. For the linguistic task, sad items with stress on the second syllable were added to prevent participants from using an emotional strategy to detect stress position. Each item was 
Table 1 Acoustic properties of the linguistic and emotional prosodic contrasts ( $S D \mathrm{~s}$ in parentheses)

\begin{tabular}{lllll}
\hline & Stressed syllable & Unstressed syllable & Angry word & Sad word \\
\hline Mean intensity $(\mathrm{dB})$ & $74.22(2.56)$ & $73.28(2.41)$ & $72.51(1.18)$ & $73.94(1.57)$ \\
Mean $\mathrm{F}_{0}(\mathrm{~Hz})$ & $239.62(54.71)$ & $219.95(36.59)$ & $255.30(13.81)$ & $202.37(35.35)$ \\
Total duration $(\mathrm{s})$ & $0.37(0.058)$ & $0.30(0.05)$ & $0.89(0.057)$ & $0.71(0.018)$ \\
Mean variation $(S D)$ in $\mathrm{F}_{0}$ & $57.53(34.29)$ & $31.98(15.30)$ & $65.88(9.88)$ & $42.66(16.47)$ \\
Mean variation $(S D)$ in intensity & $8.70(2.74)$ & $6.07(1.65)$ & $13.68(1.78)$ & $6.67(1.40)$ \\
\hline
\end{tabular}

presented ten times to each ear, resulting in a total of 120 target and 280 nontarget trials per ear. Hence, a target occurred on $30 \%$ of the trials for both the emotional and the linguistic DL tasks, and the target probability was $50 \%$ between the two ears. Task-irrelevant prosodic categories (e.g., word-initial vs. word-final stress for the emotional task) had a $50 \%$ probability of occurring.

\section{Procedure}

Each participant completed one of the two dichotic target detection tasks in a soundproof booth. Participants were instructed that they would hear a prosodic stimulus in one ear and people babbling in the other ear. They were told to ignore the babble stimulus and to decide as quickly and accurately as possible when they heard the target prosody by pressing the spacebar with the index finger. Response hands were counterbalanced across participants. Participants were instructed that they could respond while the stimulus was still playing (i.e., RTs were recorded from the onset of the stimulus). All instructions were exactly the same for both tasks, except for the description of the task-specific prosodic stimulus categories.

Each DL task started with 12 dichotic practice trials. Participants kept practicing these items until a performance level of at least $75 \%$ correct was reached. Subsequently, the experimental trials started, which encompassed a total of 400 dichotic trials (120 targets, 200 nontargets, and 80 fillers). The stimulus presentation order was (pseudo)random, with the restriction that no more than two consecutive presentations of a target were allowed.

An experimental trial started with a black fixation cross that was presented for $1,500 \mathrm{~ms}$. Subsequently, a red fixation cross and a binaural warning tone of $500 \mathrm{~Hz}$ were presented for $500 \mathrm{~ms}$, after which the dichotic stimulus was presented while the fixation cross remained red. The trial ended 2,000 ms after stimulus onset, or when participants made a response. Participants were instructed to fix their gaze on the fixation cross throughout the experiment in order to reduce eye movements, and not to blink while the fixation cross was red (i.e., when the stimuli were presented).
Stimulus presentation was controlled using E-Prime 1.2, and the stimulus materials were presented at 16-bit resolution and a 44.1-kHz sampling frequency through headphones at a comfortable intensity level.

\section{EEG recordings}

EEG was recorded from 64 tin electrodes mounted in an elastic electro-cap organized according to the International 10-20 system. EEG data were recorded with a linked mastoid physical reference and were re-referenced using an average reference. Vertical and horizontal electrooculograms (VEOG and HEOG) were recorded with two pairs of electrodes, one pair placed above and below the left eye, the other pair placed beside the two eyes. The ground electrode was applied to the sternum. The impedance of all electrodes was kept below 5 $\mathrm{k}^{\prime} \Omega$ for each participant. EEG was continuously recorded at a sampling rate of $500 \mathrm{~Hz}$, amplified, and offline digitally lowpass filtered with a cutoff frequency of $30 \mathrm{~Hz}$. Participants were seated in front of a monitor at a distance of approximately $50 \mathrm{~cm}$ in a dimly lit, electrically shielded, and soundattenuated booth.

\section{Behavioral analysis}

A repeated measures (RM) multivariate analysis of variance (MANOVA) was performed with Ear (left, right) as a withinsubjects factor and Task (emotional, linguistic) as a betweensubjects factor. The dependent measures were proportions of correct responses and the mean reaction times for correct responses.

\section{ERP analysis}

The EEG data were analyzed with Brain Vision Analyzer (version 1.05). Prior to averaging, trials with eye movement and blink artifacts were excluded from analysis. The criteria for artifact rejection within an epoch were a maximal voltage step of $50 \mu \mathrm{V}$, a maximal difference between two values in a segment of $100 \mu \mathrm{V}$, and a minimal and maximal amplitude of -100 and $100 \mu \mathrm{V}$, respectively. 
ERP epochs were computed in a 1,000-ms time window and aligned to a $100-\mathrm{ms}$ prestimulus baseline. Individual averages included at least 38 correctly detected target trials per ear. On the basis of previous literature on attentive auditory target detection (see, e.g., Fitzgerald \& Picton, 1983; Nager et al., 2001; Becker \& Reinvang, 2007), we expected to find negativities in the $\mathrm{N} 2$ range, followed by a late ( $>300 \mathrm{~ms}$ ) positive potential (LPP) including a P3b and a slow wave, when comparing targets to nontargets. Visual inspection of the ERP waves confirmed our expectations, and additionally suggested an early negativity in the N1 latency range for targets as compared to nontargets (see Fig. 2 in the Results). Although both peak and mean-amplitude analyses were considered, we decided to exclusively report mean-amplitude analyses because only the early negativity exhibited a clear peak (as can be observed in Fig. 2). For each participant, the mean amplitudes for correct target detection were computed for $100-140 \mathrm{~ms}$ (early negativity), $180-320 \mathrm{~ms}$ (N2), and 350-900 ms (LPP). Because the early negativity exhibited a clear peak, the peak latency could also be analyzed.

Due to the relatively large sample size of the present investigation, an analysis of the electrophysiological correlate of the ear advantage was possible. Participants were divided into a left-ear advantage (LEA) group and a right-ear advantage (REA) group, defined as the negative or positive difference between reaction times for correct responses for the left and right ears, respectively. This group analysis served to identify differences in ERP components depending on the laterality of the ear advantage (LEA vs. REA).

For all analyses, a set of 36 electrodes was used (see Fig. 1). For each hemisphere, the 18 electrodes were divided into six

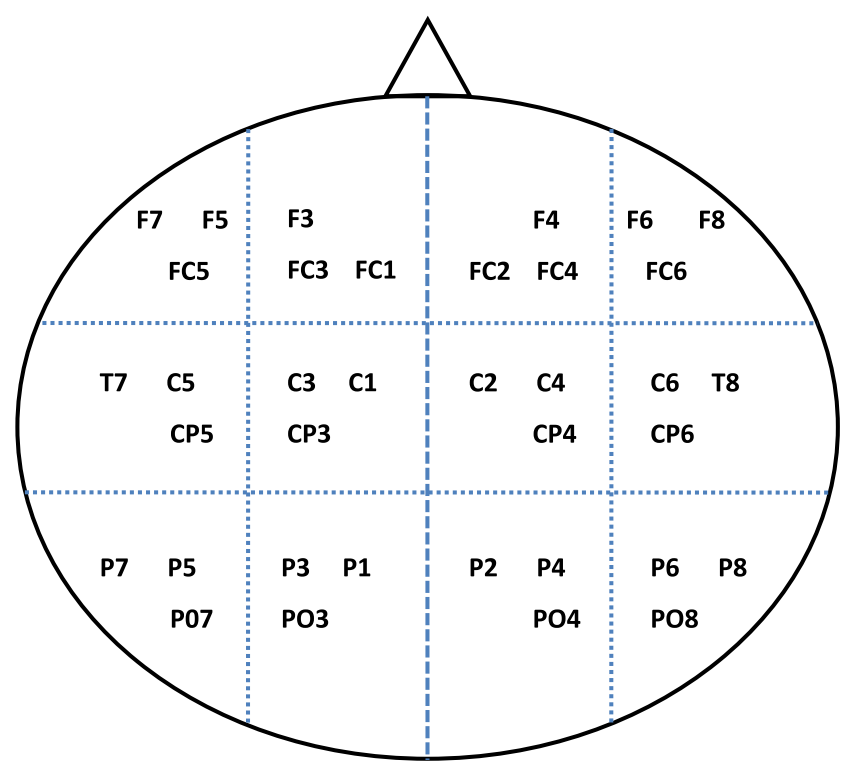

Fig. 1 Electrode array used for the experiment. Dotted lines indicate the combination of the levels of the factors Laterality (central, lateral) and Longitudinality (anterior, central, posterior) for each hemisphere (left, right) regions of interest (ROIs) comprising three electrodes each. Topographic effects were then analyzed using two factors: Laterality (left hemisphere, proximal electrodes: F3, FC3, $\mathrm{FC} 1, \mathrm{C} 3, \mathrm{C} 1, \mathrm{CP} 3, \mathrm{P} 3, \mathrm{PO} 3, \mathrm{P} 1$; lateral electrodes: F7, F5, FC5, T7, C5, CP5, P7, P5, PO7; right hemisphere, proximal electrodes: F4, FC4, FC2, C4, CP4, C2, P4, PO4, P2; lateral electrodes: F8, F6, FC6, T8, C6, CP6, P8, P6, PO8) and Longitudinality (left hemisphere, frontal electrodes: F7, F5, FC5, F3, FC3, FC1; central electrodes: T7, C5, CP5, C3, C1, $\mathrm{CP} 3$; posterior electrodes: $\mathrm{P} 7, \mathrm{P} 5, \mathrm{PO} 7, \mathrm{P} 3, \mathrm{P} 1, \mathrm{PO} 3$; right hemisphere, frontal electrodes: F8, F6, FC6, F4, FC4, FC2; central electrodes: T8, C6, CP6, C4, C2, CP4; posterior electrodes: $\mathrm{P} 8, \mathrm{P} 6, \mathrm{PO} 8, \mathrm{P} 4, \mathrm{P} 2, \mathrm{PO} 4)$.

For the main analyses, a MANOVA was conducted using PASW statistics with Ear (left, right), Hemisphere (left, right), Laterality (proximal, lateral), and Longitudinality (frontal, central, posterior) as within-subjects factors, Task (linguistic, emotional) as a between-subjects factor, and mean amplitude as the dependent variable (for the N1 component, latency was additionally analyzed as the dependent variable). An additional MANOVA was performed using exactly the same factors, but with the additional factor EA Group used to investigate the electrophysiological correlates of the behavioral ear advantage. Greenhouse-Geisser corrected $p$ values are reported.

\section{Results}

Behavioral results

The behavioral results can be found in Table 2. RM MANOVA suggested that the emotional task was easier than the linguistic task, as indicated by a main effect of task $[F(2$, $\left.71)=91.54, p<.001, \eta_{p}^{2}=.72\right]$. Follow-up univariate tests confirmed that both reaction times were faster $[F(1,71)=$ $\left.180.91, p<.001, \eta_{\mathrm{p}}{ }^{2}=.72\right]$ and accuracy higher $[F(1,71)=$ $\left.57.84, p<.001, \eta_{\mathrm{p}}^{2}=.45\right]$ for the emotional than for the linguistic task.

In sum, the behavioral results suggest that the emotional prosody task was easier than the linguistic prosody task, but no statistically reliable ear advantage was observed.

Table 2 Behavioral results for the experimental conditions ( $S D$ in parentheses)

\begin{tabular}{llllll}
\hline & \multicolumn{2}{l}{ Linguistic task } & & \multicolumn{2}{l}{ Emotional task } \\
\cline { 2 - 3 } \cline { 5 - 6 } & Left ear & Right ear & & Left ear & \multicolumn{2}{l}{ Right ear } \\
\hline Accuracy (\% correct) & $88(0.10)$ & $86(0.11)$ & & $99(0.02)$ & $99(0.01)$ \\
Reaction time (ms) & $1,016(178)$ & $1,025(191)$ & $591(83)$ & $598(76)$ \\
\hline
\end{tabular}


Electrophysiological results: task effects

The ERP waves of the early negativity, N2, and LPP are visualized in Fig. 2. Since the present article is focused on task effects, in the results below, only task-related effects and the most complete interactions are discussed. For the qualified main effects and simpler interactions and non-task-related effects, we refer the reader to the supplementary information.

Early negativity The RM MANOVA for mean amplitude revealed a Hemisphere $\times$ Ear $\times$ Longitudinality $\times$ Task interaction $\left[F(2,142)=3.34, p<.05, \eta_{\mathrm{p}}{ }^{2}=.05\right]$. Following up with separate ANOVAs for each level of longitudinality revealed that at frontal sites, the left ear elicited a larger negativity than did the right for the emotional task in both hemispheres, whereas for the linguistic task, each ear elicited a larger negativity in the contralateral hemisphere $[F(1,72)=$ $\left.6.25, p<.05, \eta_{\mathrm{p}}^{2}=.08\right]$, whereas at central $[F(1,72)=$ $\left.0.2, p>.05, \eta_{\mathrm{p}}^{2}=.003\right]$ and posterior sites $[F(1,72)=$ $\left.0.1, p>.05, \eta_{\mathrm{p}}{ }^{2}=.001\right]$, no three-way interaction emerged.

An identical MANOVA with latency as the dependent variable revealed a Hemisphere $\times$ Ear $\times$ Task interaction $\left[F(1,71)=5.93, p<.05, \eta_{\mathrm{p}}{ }^{2}=.08\right]$, with shorter latencies in the contralateral hemisphere for each ear for the emotional task, whereas for the linguistic task, latencies were shorter in the hemisphere ipsilateral to each ear.

In sum, at frontal sites the left ear elicited a stronger negativity than did the right ear in both hemispheres for the emotional task, whereas for the linguistic task, each ear elicited a larger negativity in the contralateral hemisphere. Finally, latencies were shorter in the hemisphere contralateral to the stimulated ear for the emotional task, whereas for the linguistic task, latencies were shorter in the ipsilateral hemisphere.

N2 The MANOVA for mean amplitude revealed a Longitudinality $\times$ Task interaction $[F(2,142)=19.74, p<$
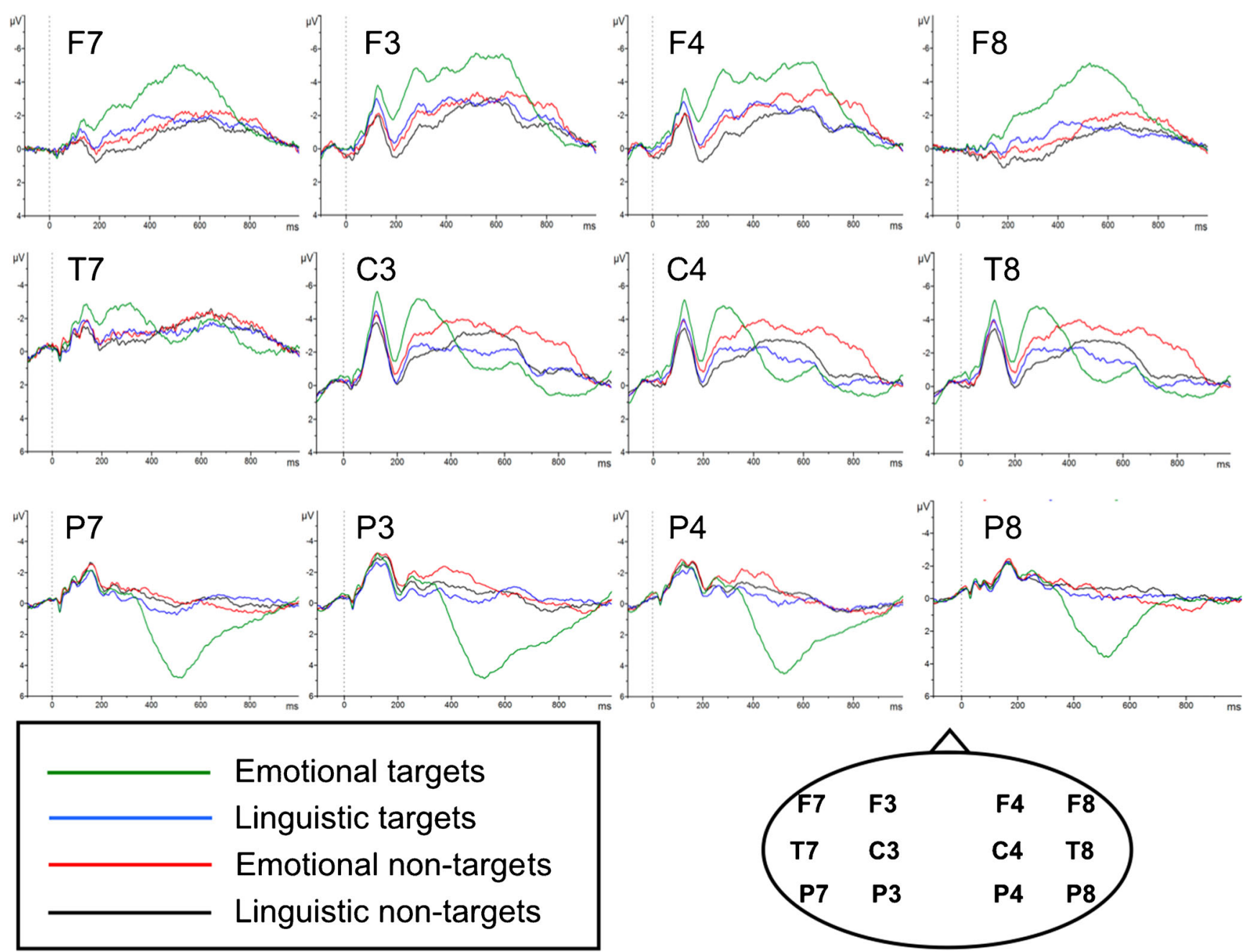

Fig. 2 Grand averages for the perception of emotional (targets green, nontargets red in the electronic version) and linguistic (targets blue, nontargets black in electronic version) prosody for a representative set of electrodes used in the analyses. Averages are shown for a 1,000-ms time window post-target-onset 
$\left..001, \eta_{\mathrm{p}}{ }^{2}=.22\right]$. Following up with separate ANOVAs for each level of longitudinality revealed a larger N2 amplitude for the emotional task than for the linguistic task at frontal sites $\left[F(1,72)=13.24, p=.001, \eta_{\mathrm{p}}{ }^{2}=.16\right]$, a trend for the emotional task to elicit a greater negativity than the linguistic task at central sites $\left[F(1,72)=3.76, p=.057, \eta_{\mathrm{p}}{ }^{2}=.05\right]$, and a greater negativity for the linguistic than for the emotional task at posterior sites $\left[F(1,72)=6.04, p<.05, \eta_{\mathrm{p}}^{2}=.08\right]$.

In sum, the emotional task elicited a larger negativity than did the linguistic task at frontocentral sites, whereas the linguistic task elicited a larger negativity than did the emotional task at posterior sites.

LPP The MANOVA for mean amplitude revealed a Hemisphere $\times$ Longitudinality $\times$ Task interaction $[F(2,144)=6.23$, $\left.p<.01, \eta_{\mathrm{p}}{ }^{2}=.08\right]$. Following up the interaction with separate ANOVAs for each level of longitudinality revealed a significant Hemisphere $\times$ Task interaction at posterior sites $[F(1,72)=$ $\left.8.28, p<.01, \eta_{\mathrm{p}}{ }^{2}=.10\right]$, with a clear positivity for the emotional task that was larger for the left than for the right hemisphere, whereas for the linguistic task, we found a much smaller positivity that was larger for the right than for the left hemisphere. For frontal and central sites, no significant Task $\times$ Hemisphere interaction emerged $(p>.05)$.

Furthermore, we observed a Hemisphere $\times$ Laterality $\times$ Task interaction $\left[F(2,144)=7.65, p<.01, \eta_{\mathrm{p}}{ }^{2}=.10\right]$. Follow-up ANOVAs for each task revealed a significant Hemisphere $\times$ Laterality interaction for the emotional task $\left[F(1,40)=13.03, p<.01, \eta_{\mathrm{p}}{ }^{2}=.25\right]$, with a positivity for proximal sites that was larger for the right than for the left hemisphere and a negativity for lateral sites that was larger for the right than for the left hemisphere, whereas for the linguistic task, no significant interaction was apparent $(p>.05)$.

To summarize, for the emotional task, we found a late positivity that was larger in the left than in the right hemisphere at posterior sites, and larger in the right than in the left hemisphere for proximal sites, irrespective of intrahemispheric location. However, for the linguistic task a highly reduced positivity emerged that was larger in the right than in the left hemisphere (see Fig. 2).

Electrophysiological results: ERP correlates of the ear advantage

The LEA and REA groups consisted of 46 and 28 participants, respectively. The two groups did not differ in male:female ratio, age, or task performance (for all, $p \mathrm{~s}>.05$ ). The groups showed a very large difference in the mean ear advantage $[t(1,71)=$ $-10.17, p<.001$, Cohen's $d=2.46$ ], with a very large mean LEA and REA of $-34 \mathrm{~ms}(S D=26$, Cohen's $d=-1.30)$ and $33 \mathrm{~ms}(S D=30$, Cohen's $d=1.14)$, respectively. For both groups, the ear advantage was robust (paired $t$ test for both groups: $p<.001)$. In the section below, only significant interactions with EA group are discussed (for a complete overview of the effects, please see the supplementary information).

Early negativity No significant main effects or interactions were found for the MANOVA with mean amplitude as dependent variable.

The MANOVA with peak latency as a dependent variable revealed a Hemisphere $\times$ Laterality $\times$ EA Group interaction $\left[F(1,69)=4.96, p<.05, \eta_{\mathrm{p}}{ }^{2}=.10\right]$. Follow-up MANOVAs for each group showed that for the LEA group, the two-way interaction was nonsignificant $(p>.05)$, but for the REA group, a significant interaction was apparent $[F(1,26)=11.75, p<.01$, $\left.\eta_{\mathrm{p}}{ }^{2}=.31\right]$, with shorter latencies for the left than for the right hemisphere, but only at lateral sites (see Fig. 3).

N2 No main effects or interactions were found.

$L P P$ No main effects or interactions were found.

\section{Discussion}

The goal of the present investigation was to test whether functional hemispheric specialization for prosody perception could be demonstrated. This was achieved by varying the emotional versus linguistic prosodic processing mode between participants, using identical prosodic stimuli in a DL ERP paradigm. No functional lateralization effects were observed at the behavioral or the electrophysiological level. Overall, the emotional task generated a larger response than did the linguistic task. Finally, the behavioral ear advantage correlated with hemispheric asymmetry of the early negativity latency for the REA group. The absence of functional

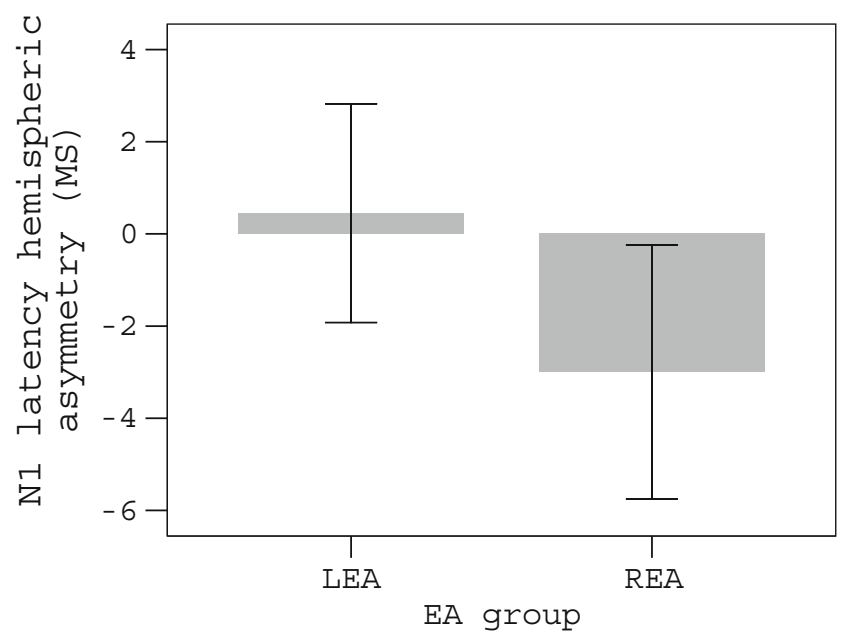

Fig. 3 Hemispheric asymmetry of the N1 peak latency (left hemisphere peak latency - right hemisphere peak latency) for lateral electrodes in the left-ear advantage (LEA) and right-ear advantage (REA) groups 
lateralization effects, task effects, and the neural correlate of the right-ear advantage will be discussed below.

Functional hemispheric specialization for prosody perception

As we outlined in the introduction, recent meta-analytic evidence suggests a relative right-hemispheric specialization for emotional prosody perception, whereas linguistic prosody perception is under bilateral control (Witteman et al., 2011). Hemispheric specialization on the one hand has been proposed to result from a non-prosody-specific righthemispheric advantage in the processing of relevant acoustic cues (i.e., the cue-dependent lateralization hypotheses), whereas the functional lateralization hypothesis, on the other hand, proposes that the left and right hemisphere are specialized in the processing of the functional categories of emotion versus linguistic prosody, respectively. The dynamic dualpathway model of Friederici and Alter (2004) further specifies that in the case of linguistic prosody perception, lateralization may depend on the degree to which prosody is bound to segmental structure, with linguistic prosody that is relatively closely bound to segments (such as metical stress, as used in the present investigation) being left-lateralized, and linguistic prosody that is not closely bound to the segmental structure (such as boundary marking) being right-lateralized. Note that the functional hypothesis requires that an abstract, categorical level of processing be reached (in order to deduce the functional category of prosodic information) before functional hemispheric specialization can take place.

As was outlined in the introduction, recent models have proposed a three-stage process of (emotional) prosody perception (Brück, Kreifelts, \& Wildgruber, 2011; Kotz \& Paulmann, 2011), including (1) extraction of acoustic properties in the primary auditory cortex, (2) integration of acoustic properties into a meaningful suprasegmental representation in the associative auditory cortex, and (3) explicit evaluation in frontal cortical areas. It has been suggested that it takes at least $100 \mathrm{~ms}$ to reach the first more-abstract stage (Stage 2) level of processing (Schirmer \& Kotz, 2006). Therefore, assuming a strictly serial model, functional lateralization effects are expected to be present in the electrophysiological signal $100 \mathrm{~ms}$ after the presentation of a prosodic stimulus. However, it seems likely that the prosody perception process is dynamic, allowing for the possibility of task demands modulating (hemispheric specialization for) earlier stages of prosodic perception (e.g., Brechmann \& Scheich, 2005) through topdown modulation.

Since both acoustic and functional properties have been hypothesized to influence hemispheric specialization for prosody perception (Pell, 1998), it is necessary to vary the functional task demands of a prosody perception task while keeping acoustics constant and to observe whether the hemispheric asymmetry of neural activity shifts in order to test the functional hemispheric lateralization hypothesis. Therefore, in the present study we instructed one group of participants to categorize the emotional and another group to categorize the linguistic prosodic dimension of the same, bidimensional prosodic stimuli. Furthermore, to maximize power to detect hemispheric specialization effects, we presented stimuli in a DL paradigm with a relatively large sample of participants, as compared to previous research. Indeed, using $\mathrm{G}^{*}$ Power (Faul, Erdfelder, Lang, \& Buchner, 2007) we calculated that even a small-to-moderate effect of the critical Ear $\times$ Task interaction could be detected with sufficient $(80 \%)$ power in the present study. However, no functional hemispheric specialization effects were found at the behavioral level (as indexed by the ear advantage) or at the electrophysiological level. Two explanations seem plausible for the absence of this effect: (1) functional lateralization effects are of considerable magnitude, but the present paradigm was not able to detect the effect of interest; (2) functional lateralization effects are too small to be picked up, even by the present (relatively high-powered) study. The first explanation seems unlikely, since a standard dichotic target detection paradigm was employed in the present investigation that has frequently been used in previous studies. Furthermore, an ERP waveform was detected that is typically observed in target detection paradigms (initial negativity followed by a positivity), demonstrating the validity of the paradigm and suggesting that the present paradigm should, in principle, have been able to detect functional lateralization effects.

Concerning the second explanation, an examination of the previous evidence in favor of functional lateralization is required. Only three previous studies had the necessary design to test the functional lateralization hypothesis. First, in a behavioral study, Luks, Nusbaum, and Levy (1998) presented 32 participants with utterances that were pronounced as either a question or a statement in the DL paradigm. Participants were instructed to categorize the utterances as a question or a statement. No ear advantage was found. In a second experiment, 50 participants had to categorize the same utterances, but now emotionally (i.e., whether the utterances sounded surprised or neutral). This time, an LEA was found. The authors concluded that the ear advantage could be modulated by task demands alone. However, these results are only partially in line with the functional lateralization hypothesis, since the expected REA for the linguistic prosody categorization task was not found. Second, Wildgruber et al. (2004) contrasted discrimination of sentential focus (linguistic prosody perception) to discrimination of the expressiveness of the same stimuli (which can be argued to fall in the category of emotional prosody) in a functional imaging study including ten participants. When contrasting the two tasks directly, a cluster of activity was observed in the left dorsolateral prefrontal cortex (DLPFC) for the perception of linguistic prosody, whereas bilateral orbitofrontal activation was noted for 
the perception of emotional prosody. Although this seems to provide evidence in favor of a greater left- than righthemispheric contribution to linguistic prosody perception, no formal tests on hemispheric asymmetry were performed, leaving it unclear whether the left DLPFC was indeed significantly more active than its right-hemispheric counterpart. Also, the expected right-lateralized activity for the emotional task was not found. Finally, a recent ERP study using the cross-splicing paradigm with a sample size of 20 participants also failed to find (functional) hemispheric specialization effects for emotional versus linguistic prosody perception (Paulmann et al., 2012). Therefore, on the basis of the scarce evidence to date, we conclude that the evidence for functional lateralization in prosody perception is weak, and future high-powered studies that manipulate the function of prosody independently of acoustics while measuring neural activity over the two hemispheres will be required to clarify this issue.

\section{Task effects}

Although no significant interaction between task demands and hemispheric asymmetry was found, emotional task demands enhanced the amplitude of all ERP components across both hemispheres, relative to the linguistic task. Two explanations may account for these effects. On the one hand, directing attention to the emotional dimension of the stimuli might have enhanced processing (or the emotional dimension might have interfered with linguistic processing during the linguistic task). Indeed, previous studies have shown evidence for the enhancement of ERP amplitudes by emotional task demands (e.g., for the LPP, see Hajcak, Moser, \& Simons, 2006; Naumann, Bartussek, Diedrich, \& Laufer, 1992), which was interpreted as prioritized processing of emotional information. On the other hand, task differences could be explained by differences in task difficulty, since the emotional task was easier than the linguistic task in the present investigation. Indeed, previous ERP studies have shown enhanced amplitudes when the difficulty of a task is reduced (e.g., for the LPP, see Gaál, Csuhaj, \& Molnár, 2007; Molnár, 1999; but see Combs \& Polich, 2006, for conflicting evidence). These two explanations might be nonmutually-exclusive, since the processing of emotional information might be easier than linguistic processing because of its comparatively large salience. Future studies that vary emotional versus nonemotional task demands while controlling for difficulty (or vice versa) will be needed to shed light on this issue.

Finally, early negativity amplitudes were larger across both hemispheres for the left ear than for the right for the emotional task, whereas the reverse was true for the linguistic task. Although it is tempting to interpret this interaction as an electrophysiological correlate of the LEA and REA, respectively, these effects were unrelated to the behavioral EA effects.

\section{Electrophysiological correlates of the EA}

Although we did not find an overall EA for linguistic or emotional prosody perception, the relatively large sample size of the present investigation permitted an additional analysis to explore the electrophysiological correlates of the EA. Participants could be divided into an REA or an LEA group, independently of whether the emotional or the linguistic task was performed, allowing for an analysis of possible underlying electrophysiological determinants of the EA. This analysis revealed that only hemispheric asymmetry in the latency of the early negativity for lateral sites could account for REAs, with REAs being associated with shorter latencies in the left than in the right hemisphere. For LEAs, however, such a latency difference was not found.

The "structural model" of the EA proposed by Kimura (see Della Penna et al., 2007) suggests that EAs arise in the DL situation because ipsilateral neural routes from the ear to the hemisphere are suppressed. When the specialized hemisphere is ipsilateral to the stimulated ear, the signal has to be transferred over the corpus callosum to reach it, and the reaction time is delayed. The present results are in line with the structural model, since REAs (but not LEAs) were associated with a delayed response of the ipsilateral hemisphere. Our results suggest that REAs can be explained by relatively early specialization at the level of the auditory cortex of the contralateral hemisphere, since the locus of the auditory $\mathrm{N} 1$ has been suggested to lie in the auditory cortex (Sandmann et al., 2007). Such early ERP correlates of the EA have been reported before in the literature (Eichele, Nordby, Rimol, \& Hugdahl, 2005; but for conflicting evidence, see Greenwald \& Jerger, 2003). However, other studies have found later ERP components to be correlated with the EA (Ahonniska, Cantell, Tolvanen, \& Lyytinen, 1993; Erhan et al., 1998; Teder, Alho, Reinikainen, \& Näätänen, 1993). As was suggested by Erhan et al., the N1 asymmetry in favor of the left hemisphere might not be related to prosodic processing, but rather to the detection of phonemes. Thus, although this is speculative, the REAs found in the present study may represent relatively early left-hemispheric specialization for phonetic processing during prosody processing.

\section{Strengths and weaknesses}

The present study is the first to systematically test the functional lateralization hypothesis of prosody perception using both behavioral evidence (EAs) and ERPs, and with the highest statistical power to date. Despite this relatively high statistical power, we failed to find evidence for functional lateralization, which we have argued may reflect the modest 
magnitude of this effect. The task differences found were confounded with a task difficulty effect, rendering it uncertain whether these differences reflected differences in the linguistic versus emotional mode of processing or the differential difficulty of the tasks. Finally, we included only negative emotions, restricting inferences about emotion effects to negative valence.

\section{Conclusion}

The present investigation did not show evidence for the functional lateralization hypothesis of prosody perception, despite relatively high statistical power, suggesting that functional lateralization effects are small. Evidence was found in favor of the idea that REAs can be explained by a speed-ofprocessing advantage of the contralateral auditory cortex, in line with the structural model of dichotic listening.

Author note We thank Jos Pacilly for doing programming work for this study.

\section{Appendix: Pseudowords used in the experiments}
Dundon
Kaldun
Duldin
Kanpal
Daldan
Kuldul
Kuldil
Duldun
Dalpal
Kulpul

\section{References}

Ahonniska, J., Cantell, M., Tolvanen, A., \& Lyytinen, H. (1993). Speech perception and brain laterality: The effect of ear advantage on auditory event-related potentials. Brain and Language, 45, 127-146.

Becker, F., \& Reinvang, I. (2007). Successful syllable detection in aphasia despite processing impairments as revealed by event-related potentials. Behavioral and Brain Functions, 3, 6. doi:10. 1186/1744-9081-3-6

Brechmann, A., \& Scheich, H. (2005). Hemispheric shifts of sound representation in auditory cortex with conceptual listening. Cerebral Cortex, 15, 578-587.

Briggs, K. E., \& Martin, F. H. (2009). Affective picture processing and motivational relevance: Arousal and valence effects on ERPs in an oddball task. International Journal of Psychophysiology, 72, 299-306.

Brück, C., Kreifelts, B., \& Wildgruber, D. (2011). Emotional voices in context: A neurobiological model of multimodal affective information processing. Physics of Life Reviews, 8, 383-403.
Combs, L. A., \& Polich, J. (2006). P3a from auditory white noise stimuli. Clinical Neurophysiology, 117, 1106-1112.

Cutler, A. (2005). Lexical stress. In D. B. Pisoni \& R. E. Remez (Eds.), The handbook of speech perception (pp. 264-289). Oxford, UK: Blackwell.

Davidson, R. J., \& Hugdahl, E. K. (Eds.). (1995). Brain asymmetry. Cambridge, MA: MIT Press.

Della Penna, S., Brancucci, A., Babiloni, C., Franciotti, R., Pizzella, V., Rossi, D., \& Romani, G. L. (2007). Lateralization of dichotic speech stimuli is based on specific auditory pathway interactions: Neuromagnetic evidence. Cerebral Cortex, 17, 2303-2311. doi:10. 1093/cercor/bhl139

Eichele, T., Nordby, H., Rimol, L. M., \& Hugdahl, K. (2005). Asymmetry of evoked potential latency to speech sounds predicts the ear advantage in dichotic listening. Cognitive Brain Research, 24, 405-412.

Erhan, H., Borod, J. C., Tenke, C. E., \& Bruder, G. E. (1998). Identification of emotion in a dichotic listening task: Event-related brain potential and behavioral findings. Brain and Cognition, 37, 286-307.

Faul, F., Erdfelder, E., Lang, A.-G., \& Buchner, A. (2007). G*Power 3: A flexible statistical power analysis program for the social, behavioral, and biomedical sciences. Behavior Research Methods, 39, 175-191. doi:10.3758/BF03193146

Fitzgerald, P. G., \& Picton, T. W. (1983). Event-related potentials recorded during the discrimination of improbable stimuli. Biological Psychology, 17, 241-276.

Friederici, A. D., \& Alter, K. (2004). Lateralization of auditory language functions: A dynamic dual pathway model. Brain and Language, 89, 267-276.

Gaál, Z. A., Csuhaj, R., \& Molnár, M. (2007). Age-dependent changes of auditory evoked potentials-Effect of task difficulty. Biological Psychology, 76, 196-208.

Greenwald, R. R., \& Jerger, J. (2003). Neuroelectric correlates of hemispheric asymmetry: spectral discrimination and stimulus competition. Journal of the American Academy of Audiology, 14, 434-443.

Grimshaw, G. M., Kwasny, K. M., Covell, E., \& Johnson, R. A. (2003). The dynamic nature of language lateralization: Effects of lexical and prosodic factors. Neuropsychologia, 41, 1008-1019.

Hajcak, G., Moser, J. S., \& Simons, R. F. (2006). Attending to affect: Appraisal strategies modulate the electrocortical response to arousing pictures. Emotion, 6, 517-522.

Kotz, S. A., \& Paulmann, S. (2011). Emotion, language, and the brain. Language and Linguistics Compass, 5, 108-125.

Luks, T. L., Nusbaum, H. C., \& Levy, J. (1998). Hemispheric involvement in the perception of syntactic prosody is dynamically dependent on task demands. Brain and Language, 65, 313-332.

Molnár, M. (1999). The dimensional complexity of the P3 event-related potential: Area-specific and task-dependent features. Clinical Neurophysiology, 110, 31-38.

Nager, W., Rosenthal, O., Bohrer, I., Teder-Sälejärvi, W. A., \& Münte, T. F. (2001). Human event-related potentials and distraction during selective listening. Neuroscience Letters, 297, 1-4.

Naumann, E., Bartussek, D., Diedrich, O., \& Laufer, M. (1992). Assessing cognitive and affective information processing functions of the brain by means of the late positive complex of the eventrelated potential. Journal of Psychophysiology, 6, 285-298.

Oldfield, R. C. (1971). The assessment and analysis of handedness: The Edinburgh inventory. Neuropsychologia, 9, 97-113. doi:10.1016/ 0028-3932(71)90067-4

Paulmann, S., Jessen, S., \& Kotz, S. (2012). It's special the way you say it: An ERP investigation on the temporal dynamics of two types of prosody. Neuropsychologia, 50, 1609-1620.

Pell, M. D. (1998). Recognition of prosody following unilateral brain lesion: Influence of functional and structural attributes of prosodic contours. Neuropsychologia, 36, 701-715. 
Polich, J., \& Bondurant, T. (1996). P300 sequence effects, probability, and interstimulus interval. Physiology and Behavior, 61, 843-849.

Sandmann, P., Eichele, T., Specht, K., Jäncke, L., Rimol, L. M., Nordby, H., \& Hugdahl. (2007). Hemispheric asymmetries in the processing of temporal acoustic cues in consonant-vowel syllables. Restorative Neurology and Neuroscience, 25, 227-240.

Scherer, K. R. (2003). Vocal communication of emotion: A review of research paradigms. Speech Communication, 40, 227-256.

Schirmer, A., \& Kotz, S. A. (2006). Beyond the right hemisphere: Brain mechanisms mediating vocal emotional processing. Trends in Cognitive Sciences, 10, 24-30.

Sussman, E., Winkler, I., Huotilainen, M., Ritter, W., \& Näätänen, R. (2002). Top-down effects can modify the initially stimulusdriven auditory organization. Cognitive Brain Research, 13, 393-405.

Teder, W., Alho, K., Reinikainen, K., \& Näätänen, R. (1993). Interstimulus interval and the selective-attention effect on auditory ERPs: "N1 enhancement" versus processing negativity. Psychophysiology, 30, 71-81.
Van Lancker, D. (1980). Cerebral lateralization of pitch cues in the linguistic signal. Papers in Linguistics: International Journal of Human Communication, 13, 201-277.

Van Lancker, D., \& Sidtis, J. (1992). The identification of affectiveprosodic stimuli by left- and right-hemisphere-damaged subjects: All errors are not created equal. Journal of Speech and Hearing Research, 35, 963-970.

Wildgruber, D., Hertrich, I., Riecker, A., Erb, M., Anders, S., Grodd, W., \& Ackermann, H. (2004). Distinct frontal regions subserve evaluation of linguistic and emotional aspects of speech intonation. Cerebral Cortex, 14, 1384-1389. doi:10.1093/cercor/bhh099

Witteman, J., Van Heuven, V. J., \& Schiller, N. O. (2012). Hearing feelings: A quantitative meta-analysis on the neuroimaging literature of emotional prosody perception. Neuropsychologia, 50, 2752 2763. doi:10.1016/j.neuropsychologia.2012.07.026

Witteman, J., van IJzendoorn, M. H., van de Velde, D., van Heuven, V. J., \& Schiller, N. O. (2011). The nature of hemispheric specialization for linguistic and emotional prosodic perception: A meta-analysis of the lesion literature. Neuropsychologia, 49, 3722-3738. doi:10. 1016/j.neuropsychologia.2011.09.028 